children under 10. Moreover, no Coxsackie virus was isolated and no association with infected cattle was found. ${ }^{1}$

Certain localized virus infections may cause papular eruptions, especially on the hands. Orf is an example, but this is found only when there is a direct or indirect contact with infected sheep. Even if the outbreak here were due to some other local virus infection and spread by contact with infected girls a succession of outbreaks would be more likely.

In pompholyx there are vesicles on the hands, but it is not a papulo-vesicular eruption, and the sides of the fingers and the palmar aspect of the hands are affected. Moreover, it is essentially an individual, constitutional reaction to some endogenous upset and, though more common in the summer, would not suddenly appear in $23 \%$ of a group of girls.

Dr. Warin and his colleagues consider the possibility of soap being the cause, and no doubt they have in mind certain soaps not now on the market which contained tetrachlorsalicylamide, a bacteriostatic agent. This material sensitizes the skin to sunlight, so that on exposure an eruption appears. Sensitization to sunlight may also result from contact with wild parsnip and certain other plants, but this would be an unlikely cause of the outbreaks described. The authors note that many of the lesions resembled chilblains, though they consider a sudden change of weather a very unlikely cause of such an epidemic. They name this curious condition "summer-term blains," and despite being unable to identify the cause of the disease their thorough and interesting report should help other physicians to keep a look-out for it. General practitioners and school doctors would seem to be especially well placed to add to our knowledge of it.

\section{Cerebral Abscess}

Despite the use of antibiotics and advances in surgical techniques, the operative mortality from cerebral abscess is still about $30 \%$. Thus no fewer than 17 out of 99 cases reported by E. Loeser and L. Scheinberg ${ }^{1}$ were diagnosed only at necropsy. Though its incidence has diminished in recent years, chiefly because inflammation of the middle ear is less frequent, cerebral abscess may still be difficult both to diagnose and to treat.

Many cases (about $40 \%$ ) are a sequel to mastoid infection, while those originating from chronic infections of the lung and cyanotic heart disease ${ }^{2}$ account for about $20 \%$ each. The incidence of the last group is increasing, possibly because the prognosis for these patients has improved considerably. Cerebral abscess is seen occasionally in non-cyanotic congenital heart disease, and the predisposition of patients with both types of congenital heart disease is probably due either to the passage of bacteria through a septal defect or shunt, or to the implantation of organisms in foci of necrosis in the brain. Finally, abscesses may follow frontal sinusitis, or trauma, or may be secondary to foci of infection elsewhere in the body. Scattered multiple abscesses may also occur and are almost always fatal. ${ }^{3}$

Because the patient has frequently been treated with antibiotics the source of infection is not always apparent by the time a cerebral abscess becomes manifest, though its origin is usually obvious. Abscesses arising from the ear are found in the mid-temporal and anterolateral cerebellar regions with

1 Alscp, J., Flewett, T. H., and Foster, J. R., Brit. med. F., 1960, 2, 1708. 2 Ibid., 1963, 2, 132. remarkable constancy; those secondary to inflammation of the frontal sinuses also tend to lie in relation to the source. Intracerebral extension from local infection is by direct spread, or by the passage of bacteria in thrombosed vessels. Alternatively infection travels via the blood-stream. It tends to develop beneath the cortex, especially in the distribution of the middle cerebral artery. The basal ganglia and cerebellum are seldom affected by blood-borne organisms.

Because of the use of antibiotics the early manifestations of non-suppurative encephalitis are now rarely seen, and at present the presenting symptom of cerebral abscess is likely to be headache or epilepsy. In addition symptoms related to the situation of the lesion such as dysphasia, hemianopia, hemiparesis, or alteration of intellectual function may be apparent. Apart from a brief and usually subclinical meningitis, which may occur at the onset of cerebral infection, a considerable increase in the number of cells in the cerebrospinal fluid is unusual unless the abscess has ruptured into a ventricle. This is not likely to happen early in the course of the disease, but it may take place before the diagnosis is established. Unless there is a frank meningitis the temperature and pulse and respiration rates may be unchanged; and the cerebrospinal cell count and sugar concentration may be scarcely abnormal, though the protein concentration may be slightly raised. Unfortunately an overwhelming meningitis may occasionally mask the presence of the abscess from which it arose.

The bacterial flora of cerebral abscesses is often mixed, ${ }^{4}$ and its nature is influenced by previous treatment. Though staphylococci, aerobic streptococci, paracolon bacilli, Proteus, and pneumococci are often seen, the frequency of anaerobic organisms is not always sufficiently appreciated. For example, H. S. Heineman and A. I. Braude ${ }^{j}$ report the bacteriological findings in 18 consecutive cases of brain abscess, in 16 of which anaerobes were cultured. Sir Hugh Cairns used to emphasize that though sterile abscesses occurred from time to time failure to grow organisms often meant that there was a fault in technique or lack of persistence. Heineman and Braude found that anaerobic streptococci were isolated most frequently, followed by bacteroides and actinomyces. $V$ eillonella organisms and anaerobic corynebacteria were less common pathogens. Failure to culture these organisms might be due to exposure of the specimen to air before culture, to insufficient time for anaerobic culture, or even to omitting to set up anaerobic preparations altogether.

Patients suspected of having a cerebral abscess should be investigated in a hospital where there are facilities for neuroradiology and electroencephalography. The management of these patients demands wide experience and should be undertaken only in a neurosurgical unit. There is no place for exploratory needling of the brain from within a mastoid cavity, for the inaccuracy and risks of this method can no longer be justified. Neither should the drainage of a cerebral abscess into a sinus or mastoid cavity be contemplated. After accurate localization an abscess requires repeated aspiration, with or without ultimate excision.

A cerebral abscess must be suspected in any patient with infection of the ear (acute or chronic), with congenital heart disease, or with chronic lung infection, or who has a known focus of infection, who develops headache, epilepsy, signs of meningitis, raised intracranial pressure, or focal neurological

\footnotetext{
1 Loeser, E., jun., and Scheinberg, L., Neurology, 1957, 7, 601.

2 Jayewardene, R., P., Brit. med. Ұ., 1956, 1, 787.

$s$ Levy, L. F., ibid., 1963, 1, 1455 .

- McFarlan, A. M., ibid., 1943, 2, 643.

- Meineman, H. S., and Braude, A. I., Amer. F. Med., 1963, 35, 682.
} 
signs. If an abscess still cannot be found after investigation, whatever alternative diagnosis may be offered, that of cerebral abscess must remain under consideration until the patient has recovered. If the source of infection remains, or if a congenital heart lesion is still present, there is a risk of further infection, while in any inadequately treated abscess there is always a risk of its recrudescence.

The primary focus of infection must be treated together with the cerebral abscess, and meningitis demands urgent control if it is present. Nevertheless, systemic antibiotics, even in massive doses, cannot reach the contents of an abscess or affect areas of brain which lack a blood supply. They may control infection in the surrounding tissue, but an abscess can be sterilized only by evacuating its contents and replacing them with an antibiotic; alternatively the abscess and its capsule may be excised.

Epilepsy is the chief hazard to the patient who has recovered, but it can usually be controlled adequately by drugs, and possibly by excision of the capsule if this has not been done already. Other disabilities may be a sequel to damage occurring at the time of acute infection. There is certainly much more to be learnt about the diagnosis and management of cerebral abscesses before the problem can be considered to have been solved.

\section{Neonatal Thrombocytopenia and Thiazide Drugs}

Thrombocytopenia in the newborn is dangerous, though uncommon. The case fatality rate is about $14 \% .^{1}$ Recovery is usually spontaneous and takes place within one to three weeks but transfusion may be required. Most infants with thrombocytopenia are born of mothers with idiopathic thrombocytopenic purpura, and it is assumed that a humoral or immune factor is transmitted to the foetus across the placenta. $^{2}$ In about $20 \%$ of cases of neonatal thrombocytopenia, however, the mother appears to be haematologically normal and an immune mechanism analogous to that for sensitization to the Rhesus factor has been suggested. In these cases both father and affected child have been shown to possess a common platelet antigen which is absent from the maternal platelets, and maternal antibodies against this platelet antigen have been demonstrated. ${ }^{3}$

A third mechanism for neonatal thrombocytopenia has been suggested by S. U. Rodriguez and his colleagues, ${ }^{4}$ and is referred to in a letter by Dr. L. Prescott at page 1438 in this week's B.M.f. Rodriguez and his co-workers report seven babies with neonatal thrombocytopenia, one of whom died, whose mothers had neither idiopathic thrombocytopenic purpura nor evidence of isoantibodies directed against the infants' platelets. These patients had been treated for toxaemia of pregnancy with chlorothiazide, hydrochloro-

1 Morris, M. B., Arch. Dis. Childh., 1954, 29, 75.

2 Harrington, W. J., Sprague, C. C., Minnich, V., Moore, C. V., Aulvin, R. C., and Dubach, R., Ann. intern. Med., 1953, 38, 433. R. C., and Dubach, R., Ann. intern. Med., 1953, 38, 433.
1964, 23. 154 .

1 Rodriguez, S. U., Leikin, S. L., and Hiller, M. C., Nerv. Engl. F. Med., 1964, 270, 881 .

- Zuckerman, A. J., and Chazan, A. A., Brit. med. F., 1958, 2, 1338.

- Jaffe, M. O., and Kierland, R. R., भ. Amer. med. Ass., 1958, 168, 2264

$?$ Posner, A. C., Amer. F. Obstet. Gynec., 1937, 34, 155.

- Mauer, A. M., DeVaux, W., and Lahey, M. E., Pediatrics, 1957, 19, 84.

- Greenwald, H. M., and Sherman, I., Amer.. Dis. Child., 1929, 38, 1245. thiazid ;, or methyclothiazide. In the absence of evidence of isoimmunization they suggested that these drugs were responsible for the haemorrhagic state in the newborn. Purpura, and often thrombocytopenia, is an occasional complication of therapy with the thiazide drugs in adults, and neutropenia and agranulocytosis have been recorded. ${ }^{5}$ We need to know more about the findings in the blood of infants of mothers treated with these oral diuretics, but the data published by Rodriguez and his colleagues are highly suggestive.

The possible mechanism of drug-induced thrombocytopenia in the newborn is of interest. A. C. Posner has described a case of quinine sensitivity producing thrombocytopenia in both mother and newborn; presumably both the drug and the antibody to it passed across the placenta. However, Rodriguez and his colleagues were unable to demonstrate a maternal antibody that might be dependent on one of the thiazide drugs for its activity. Thus either the foetus is more sensitive to the toxic effect of these drugs than the mother or there is some concentration of the drug on the foetal side of the placenta. Studies of the bone marrow of infants with neonatal thrombocytopenia also show interesting differences from the changes seen in thrombocytopenia in later life. Thrombocytopenia in adults, either idiopathic or drug-induced, is almost invariably associated with normal or increased numbers of megakaryocytes in the marrow, though these are often morphologically abnormal. In neonatal thrombocytopenia megakaryocytes are often scanty ${ }^{9}$ and this may be accompanied by other evidence of marrow damage, such as a relative diminution in the number of reticulocytes and polymorphonuclear neutrophil leucocytes. In these cases the blood may slowly return to normal over 6 to 9 months. Possibly this is the result of a relatively prolonged period of exposure of foetal marrow to those toxic agents.

\section{Induction by Association}

The recent papers in this journal by G. Negroni and his colleagues $^{12}$ and by T. M. Bell and his colleagues, ${ }^{3}$ who have discovered viruses in association respectively with human leukaemia and with a case of African lymphoma (Burkitt's tumour), have renewed interest in the problem of isolating viruses from the tumours (animal or human) which they may have caused. The cells of some virus-induced animal cancers (for example, mouse mammary carcinoma, rodent leukaemia, and chicken leukosis complex) appear to produce a causative virus which may be extracted quite simply from the tumours. Other tumours-and notably those produced by the papova group of viruses (rabbit papilloma, mouse polyoma, and simian SV 40)-produce virus much more rarely and in very small amounts. ${ }^{4}$ The papova group of viruses all contain D.N.A. (deoxyribonucleic acid), ${ }^{5}$ and the consensus of opinion seems to be that transformation of normal cells to malignant by these viruses is the result of integration of the viral D.N.A. into the genome of the infected cell-i.e., that part of the cell which reproduces itself. There are other consequences of this interaction. Viral antigens (complement-fixing ${ }^{6-8}$ ) and non-viral antigens are produced by the cells. Some of the latter act as antigens when the tumours are transplanted..$^{9-12}$ New enzymes may also be synthesized. ${ }^{13}$

The virus in tumours, whether it is present as complete 